\title{
Recommendations for the assessment and monitoring of skeletal manifestations in children with Gaucher disease
}

\author{
M. Maas • T. Hangartner • G. Mariani • K. McHugh • \\ S. Moore • G. A. Grabowski • P. Kaplan • A. Vellodi • \\ J. Yee $\cdot$ L. Steinbach
}

Published online: 19 December 2007

(C) The Author(s) 2007

\section{Introduction}

Gaucher disease (GD), the most common of the lysosomal storage disorders, results from mutations in the gene for acid $\beta$-glucosidase (glucocerebrosidase, GBA) leading to insufficient enzyme activity [1]. Glucocerebroside accumulation in the lysosomes of various cell types is responsible for the clinical manifestations of GD, which may include hepatomegaly, splenomegaly, anemia, thrombocytopenia, and bone marrow infiltration by lipid-engorged "Gaucher

M. Maas $(\bowtie)$

Department of Radiology, Suite G1-211,

Academic Medical Center, University of Amsterdam,

Meibergdreef 9,

1105 AZ Amsterdam, The Netherlands

e-mail:m.maas@amc.uva.nl

T. Hangartner

BioMedical Imaging Laboratory,

Wright State University and Miami Valley Hospital,

Dayton, OH, USA

\section{G. Mariani}

Regional Center of Nuclear Medicine, University of Pisa,

Pisa, Italy

\section{K. McHugh}

Department of Radiology,

Great Ormond Street Hospital for Children,

London, United Kingdom

\section{S. Moore}

Department of Imaging Services,

Children's Hospital of Los Angeles,

Los Angeles, CA, USA

\section{G. A. Grabowski}

Division of Human Genetics,

Cincinnati Children's Hospital Medical Center,

Cincinnati, OH, USA cells." Occurring panethnically, type 1 (non-neuronopathic) is the most prevalent form of GD, while the less frequent type 2 (acute neuronopathic) and type 3 (chronic neuronopathic) are characterized by neurological involvement. More than half of type 1 patients are diagnosed in childhood and an earlier age of onset is indicative of more severe disease due to its progressive nature.

Affecting both the marrow and mineral compartments, GD-related bone disease is the most significant cause of morbidity and long-term disability for patients. GD-related

G. A. Grabowski

Department of Pediatrics,

University of Cincinnati College of Medicine,

Cincinnati, OH, USA

P. Kaplan

Department of Pediatrics, The Children's Hospital of Philadelphia, University of Pennsylvania,

Philadelphia, PA, USA

\section{A. Vellodi}

Metabolic Unit, Great Ormond Street Hospital for Children,

London, United Kingdom

J. Yee

Global Medical Affairs, Genzyme Corporation,

Cambridge, USA

L. Steinbach

Department of Radiology, University of California San Francisco, San Francisco, CA, USA 
bone disease in children is a major cause for concern as it puts them at risk of developing irreversible and debilitating bone complications and interferes with normal growth and achievement of optimal bone mass during a critical period of growth. Timely initiation of appropriate disease management is necessary to avoid serious long-term complications such as growth retardation, osteoporosis, and fractures, and includes an initial radiological assessment and ongoing monitoring of both the bone marrow and mineral components. General guidelines for the monitoring of skeletal disease in children with type $1 \mathrm{GD}$ recommend magnetic resonance imaging (MRI) of the spine and femora; radiography of the spine (when clinically indicated), chest, pelvis, and long bones; and dual-energy X-ray absorptiometry (DXA) of the spine and hips at baseline and every 12-24 months [2, 3]. However, these recommendations require further clarification in view of the challenges associated with their use in children with GD, especially since the use of conventional radiography is limited by a high burden of radiation and lack of sensitivity and specificity.

The current standard of care for type 1 GD is enzyme replacement therapy (ERT) with imiglucerase (Cerezyme ${ }^{\circledR}$; Genzyme Corporation, Cambridge, MA, USA). Oral substrate reduction therapy with miglustat (Zavesca ${ }^{\circledR}$; Actelion, San Francisco, CA, USA) is also available for the treatment of patients with mild to moderate GD for whom ERT is not an option. The availability of therapy that may prevent or reverse GD-related bone disease heightens the need for early diagnosis and initiation of disease management in children.

A Working Group of international experts met in October 2006 to recommend evidence- and consensus-based guidelines to facilitate the assessment and monitoring of bone disease in children with type 1 or type 3 GD.

\section{Assessment of bone marrow infiltration in pediatric Gaucher patients by MRI}

Magnetic resonance imaging, the most widely used and accepted method of assessing and evaluating changes in bone marrow, is the method of choice for monitoring marrow displacement in adults with GD. This technique has also been used in children with GD to qualitatively evaluate and characterize bone marrow infiltration by Gaucher cells [4]. Advantages of MRI include widely available technology, semi-quantitative assessments, ease of measurement, high sensitivity, and lack of associated ionizing radiation.

The displacement of fatty marrow by Gaucher cells is best detected by T1-weighted MRI and appears as an abnormally low-intensity signal, which is unique compared with the triglyceride signal. Following ERT with imiglucerase in patients with GD, the responding bone marrow shows increased triglyceride content and an increase (brightening) in the bone marrow signal. T2-weighted fat saturation or short tau inversion recovery (STIR) sequences are the most sensitive for the detection of additional bone marrow complications of GD, including irreversible avascular necrosis.

Interpretation of MRI measurements with respect to the physiologic conversion of bone marrow

Neither the chronology of pediatric GD bone marrow involvement and its variations nor the impact of delayed skeletal maturation on the conversion of hematopoietic to fatty marrow in children with GD are known. Knowledge of the typical conversion patterns of bone marrow in Gaucher patients on MRI by age group as well as the availability of normative pediatric MRI data will eventually allow a more clinically useful interpretation of MRI-derived data. MR images of children with GD should be interpreted in comparison with normal physiological conversion patterns, especially in very young children whose low signal due to a high percentage of red marrow appears similar to pathologically infiltrated bone marrow. The potential contributions of therapy to observed changes in bone marrow infiltration must also be considered.

\section{Clinical interpretation of MRI measurements}

Numerous semi-quantitative scoring systems have been developed for the evaluation of bone marrow infiltration in adult Gaucher patients, including the Rosenthal score, the Düsseldorf Gaucher score, the Terk classification, the vertebra-disc-ratio, and the bone marrow burden (BMB) score [5]. Dixon Quantitative Chemical Shift Imaging (QCSI), a quantitative MR-based technique, reports the displacement of triglyceride-containing marrow by Gaucher cells as a fat fraction. The BMB scoring method is preferred over the other available scoring systems as it includes measurements of both lumbar spine and femur, key anatomical sites of Gaucher cell infiltration, and has been validated against other methods and applied in multiple studies [6, 7]. Although numerous studies on age-related distribution of fat in bone marrow in children have been performed, normal QCSI vertebral fat fractions have yet to be established for children.

Use of sedation

Children between birth and the age of 6 years usually require sedation or a general anesthetic for successful examination by MRI. In the absence of local "best practice" protocols, MRI examinations of children with GD should be carried out in accordance with the guidelines established by the American Academy of Pediatrics and the American Society of Anesthesiologists. 
${ }^{99 \mathrm{~m}}$ Tc-Sestamibi: an alternative to MRI?

${ }^{99 \mathrm{~m}} \mathrm{Tc}-$ Sestamibi accumulation in the bone marrow has been used to assess the severity of bone marrow infiltration by Gaucher cells in adults and results correlate highly with other indicators of systemic disease burden. Despite this technique's independence from the influence of bone marrow conversion, scintigraphic images are less spatially resolved than MR images, the radiation burden to the growing child is significant, and pediatric normal controls do not exist. Therefore, at present ${ }^{99 \mathrm{~m}} \mathrm{Tc}$-Sestamibi is not an acceptable alternative for assessing or monitoring bone marrow infiltration.

\section{Assessment of BMD in pediatric Gaucher patients by DXA}

Widely available, relatively inexpensive, and involving low ionizing radiation exposure, DXA is safe, noninvasive, rapid, painless, and the preferred technique for the diagnosis of osteopenia and osteoporosis in adults. DXA is routinely used to evaluate bone mineral density (BMD) in patients with GD and is capable of providing a quantitative assessment of bone involvement and monitoring changes in bone density in response to therapy. DXA is potentially useful for the evaluation of BMD in children with GD, although specific technical and practical issues need to be considered.

Technical aspects of DXA measurements in children

Due to the two-dimensional nature of DXA technology, DXA measurements are highly influenced by bone size as well as by bone age and pubertal stage. Therefore, small bone size may be misinterpreted as a low BMD. Since growth retardation, pubertal delay, and decreased body mass are common problems in pediatric GD and may distort measured BMD, these factors must be considered when interpreting the DXA-derived data. With serial measurements in the presence or absence of therapy, normal growth in a child with initially small bones should not be perceived as a false increase in BMD. Additional confounding factors for DXA scans of the hip or lumbar spine include avascular necrosis and vertebral compression fractures respectively, both of which may occur with GD. Awareness of these potential influences on BMD measurements obtained by DXA can prevent misinterpretation and improve the clinical usefulness of these data.

Clinical interpretation of DXA measurements

In adults DXA measurements are reported as T-scores, which represent the number of standard deviations from the mean
BMD of a reference group of normal gender-matched individuals in the age range (3rd decade) during which BMD peaks. T-scores are not applicable for individuals under the age of 20 years; measurements made in children are reported as age- and gender-matched Z-scores. Although Z-scores cannot diagnose osteopenia or osteoporosis, a Z-score below -2.0 or above 0.0 represents a value outside the normal range.

The clinical usefulness of DXA in children with GD depends on access to appropriate software for data analysis, normative pediatric data, and familiarity with the correct methods of reporting measurements. The major DXA manufacturers currently provide pediatric reference data of BMD versus chronological age, and gender- and ethnicity-specific reference data for healthy European and North American children have recently been published for the assessment of BMD measured by DXA [8, 9]. However, standards for adjusting BMD on the basis of bone size, pubertal stage, skeletal maturity, and body composition are also needed. Furthermore, correlations between DXA-derived data and the likelihood of developing fractures would increase the usefulness of DXA for the evaluation of BMD in children with GD.

Quantitative computed tomography: an alternative to DXA?

Unlike DXA, quantitative computed tomography (QCT) can assess bone density independently of bone size, allowing measurement of the actual volumetric density of a specific compartment and eliminating the potentially confounding factors of growth retardation and pubertal delay observed in some pediatric Gaucher patients. However, few pediatric studies using this technique and no standard analyses are available, and QCT of the spine results in considerable radiation exposure. Therefore, QCT currently cannot be recommended for use in children.

\section{Recommendations}

We recommend the following guidelines as appropriate for monitoring skeletal manifestations in children with type 1 or type 3 GD.

Performing measurements with MRI

1. Perform assessments at centers with MRI expertise in children.

2. Assess anatomical sites including a substantial part of the preferably axial bone marrow compartment (femur, pelvis, and spine) at baseline and at least every 2 years, ideally annually.

3. T1 and either T2 or STIR sequences are recommended for routine evaluation, and STIR sequences are recommended to detect complications. 
Clinical interpretation of MRI measurements

1 Magnetic resonance images of children with GD should be interpreted in comparison with normal physiological conversion patterns.

2. The potential contributions of therapy to observed changes in bone marrow infiltration must be considered.

Use of sedation for MRI measurements

The American Academy of Pediatrics and the American Society of Anesthesiologists provide guidance to facilitate the administration of sedation, if needed, in the safest manner possible.

\section{Performing measurements with DXA}

1. Perform assessments at centers with DXA expertise in children and where normal pediatric data are available.

2. Assess lumbar spine and proximal femur and the entire body at baseline and annually thereafter, provided that normal pediatric reference standards are available.

3. Perform serial scans on the same DXA machine and analyze with the appropriate pediatric option.

\section{Clinical interpretation of DXA measurements}

Dual-energy X-ray absorptiometry measurements from children (males or females $<20$ years of age) with GD should be interpreted and reported in accordance with the recommendations and caveats provided by the International Society for Clinical Densitometry [10]:

1. Z-scores must be used; T-scores should not be used in children.

2. Z-scores must be interpreted using the best available pediatric databases of age-, gender-, and ethnicitymatched controls. Reports should cite the reference database used.

3. Terminology such as "low bone density for chronologic age" or "below the expected range for age" may be used if the Z-score $<-2.0$.

4. The diagnosis cannot be made on the basis of densitometric criteria alone.

5. The value of BMD to predict the likelihood of fractures in children has not been clearly determined.

6. No agreement exists on standards for adjusting BMD for factors such as bone size, pubertal stage, skeletal maturity, and body composition. If adjustments are made, they should be clearly stated in the report.

7. Deviation from standard adult analysis protocols, such as use of low-density software and manual adjustment of the region of interest, should be stated in the report for the accurate comparison of DXA-derived data.

Role of conventional radiography

Although widely available and relatively inexpensive, we do not recommend the routine or exclusive use of conventional radiography due to its high burden of radiation and lack of sensitivity and specificity.

Acknowledgements Support for the meeting was provided by Genzyme Corporation. The opinions and recommendations of this article do not necessarily reflect the opinion of the company. The authors acknowledge assistance with preparation of the manuscript from Carolyn Sawyer, PhD (Genzyme Corporation).

Open Access This article is distributed under the terms of the Creative Commons Attribution Noncommercial License which permits any noncommercial use, distribution, and reproduction in any medium, provided the original author(s) and source are credited.

\section{References}

1. Beutler E, Grabowski GA. Gaucher disease. In: Scriver CR, Beaudet AL, Sly WS, et al., editors. The Online Metabolic and Molecular Bases of Inherited Disease. New York, NY: McGraw-Hill; 2006: 3635-3668.

2. Charrow J, Andersson HC, Kaplan P, et al. Enzyme replacement therapy and monitoring for children with type 1 Gaucher disease: consensus recommendations. J Pediatr 2004; 144: 112-120.

3. Baldellou A, Andria G, Campbell PE, et al. Paediatric nonneuronopathic Gaucher disease: recommendations for treatment and monitoring. Eur J Pediatr 2004; 163: 67-75.

4. El-Beshlawy A, Ragab L, Youssry I, et al. Enzyme replacement therapy and bony changes in Egyptian paediatric Gaucher disease patients. J Inherit Metab Dis 2006; 29: 92-98.

5. Vom Dahl S, Poll L, Di Rocco M, et al. Evidence-based recommendations for monitoring bone disease and the response to enzyme replacement therapy in Gaucher patients. Curr Med Res Opin 2006; 22: 1045-1064.

6. Robertson PL, Maas M, Goldblatt J. Semiquantitative assessment of skeletal response to enzyme replacement therapy for Gaucher's disease using the bone marrow burden score. Am J Roentgenol 2007; 188: 1521-1528.

7. de Fost M, Hollak CE, Groener JE, et al. Superior effects of highdose enzyme replacement therapy in type 1 Gaucher disease on bone marrow involvement and chitotriosidase levels: a 2-center retrospective analysis. Blood 2006; 108: 830-835.

8. Ward KA, Ashby RL, Roberts SA, Adams JE, Zulf MM. UK reference data for the Hologic QDR Discovery dual-energy x ray absorptiometry scanner in healthy children and young adults aged 6-17 years. Arch Dis Child 2007; 92: 53-59.

9. Kalkwarf HJ, Zemel BS, Gilsanz V, et al. The Bone Mineral Density in Childhood Study (BMDCS): bone mineral content and density according to age, sex and race. J Clin Endocrinol Metab 2007; 92: 2087-2099.

10. Lewiecki EM, Binkley N, Bilezikian JP, Kendler DL, Leib ES, Petak SM. Official positions of the International Society for Clinical Densitometry. Osteoporos Int 2006; 17: 1700-1701. 